\title{
Isolation and chromosomal localization of a new human retinoblastoma binding protein 2 homologue 1a (RBBP2H1A)
}

\author{
Vladimir Kashuba ${ }^{1,2,3}$, Alexei Protopopov ${ }^{1,2,4}$, Raf Podowski ${ }^{2}$, Rinat Gizatullin², \\ Jingfeng Li ${ }^{1}$, George Klein ${ }^{1}$, Claes Wahlestedt ${ }^{2}$ and Eugene Zabarovsky ${ }^{1,2,5}$

\begin{abstract}
${ }^{1}$ Microbiology and Tumor Biology Center; ${ }^{2}$ Center for Genomics Research, Karolinska Institute, Stockholm, Sweden; ${ }^{3}$ Institute of Molecular Biology and Genetics, Ukrainian Academy of Sciences, Kiev, Ukraine; ${ }^{4}$ Institute of Cytology and Genetics, Russian Academy of Sciences, N ovosibirsk; ${ }^{5}$ Engel hardt Institute of Molecular Biology, Russian Academy of Science, Moscow, Russia
\end{abstract}

Using a Notl linking clone NR-025 as a probe, we isolated a novel putative member of the RB binding protein family, namely a human retinoblastoma binding protein 2 homologue (RBBP2H1A). The maximal open reading frame encodes a protein of 1681 amino acids. Homology analysis indicated that the predicted product has an overall 56\% amino acid identity to RBBP2, which plays an important role in RB tumor suppressor regulation. Many extended regions are $100 \%$ identical in amino acids sequences. The degree of nucleotide identity is lower. The structure prediction analysis identified three DNA-binding zinc finger domains and two bipartite nuclear localization signals. Northern expression analysis revealed expression in all tissues; however, the level of expression significantly varied between tissues. The highest level of expression was detected in testis and the lowest in skeletal muscle. The mRNA sizes corresponding to two major products are around $6 \mathrm{~kb}$ and $7 \mathrm{~kb}$. Using fluorescence in situ hybridization, we mapped the gene to chromosomal band 1q32.1. European Journal of Human Genetics (2000) 8, 407-413.

Keywords: Notl-linking clone; molecular cloning; human; gene mapping; sequence alignment; fluorescence in situ hybridization

\section{Introduction}

Several years ago, we proposed an approach to combine physical and gene mapping methods to characterize large regions of human chromosomes ${ }^{1,2}$ and Notl linking and jumping clones were proposed as framework markers. We then developed simplified new procedures for jumping and linking library construction and made a number of chromosome 3 specific and total human Notl linking libraries. ${ }^{3}$

One thousand Notl linking clones from the chromosome 3 specific grids were partially sequenced and were found to represent 249 unique clones. Among these, we carefully analyzed 150 clones. $^{4}$ The results confirmed previous sugges-

Correspondence: Dr VI Kashuba, Tel: +468728 6737; Fax: +4683194 70; E-mail: vlakas@ki.se; and Dr ER Zabarovsky, Tel: +4687286750; Fax: +4683194 70; E-mail: eugzab@ki.se; both at Microbiology and Tumor Biology Center and Center for Genomics Research, Karolinska Institute, S-171 77, Stockholm, Sweden. Received 24 September 1999; revised 10 January 2000; accepted 13 January 2000 tions from us and others that: (i) Notl sites are frequently associated with human genes and (ii) that the sequence data generated can serve as an framework for joining and closing of other types of maps. ${ }^{4-7}$

We therefore decided to sequence all Notl sites in the entire human genome in the search for the new genes and to generate framework markers. The results of a pilot study, consisting of about $10 \%$ of the entire clone collection, have been analysed and will be presented elsewhere. Our analysis showed that a surprisingly large number (more than 40\%) of Notl clones have identity with ESTs. It can be nevertheless being concluded that data from our Notl project are complementary to data obtained from various EST projects. We have thus confirmed the opinion that Notl sites are located predominantly at the 5 ' end of genes while the majority of the EST clones have obviously been produced with oligo dT primers resulting in over-representation of the $3^{\prime}$ end of genes. $^{8,9}$ 
Our Notl project on the entire human genome has al ready resulted in the discovery of many new genes. Here we report on a novel putative member of the RB binding protein family, namely a human retinoblastoma binding protein 2 homologue RBBP2H1A.

\section{Materials and methods}

Construction of Notl linking libraries was described previously. ${ }^{3}$

Sequencing was done using $A B \mid 377$ sequencers according to standard procedures. Homology searches were performed using blastx and blastn programs at the NCBI server. ${ }^{10,11}$

Search of protein profiles against the PROSITE-library was done at the ISREC server (http://www.isrec.isb-sib.ch/ software/PFSCAN |form.html).

Eukaryotic promoters prediction by neural network (NNPP) was done at the BCM server (http://dot.imgen .bcm.tmc.edu:9331/seq-search/gene-search.html). The hybridization with MTN northern filters (Clontech, Palo Alto, CA, USA) was done according to the manufacturer's protocols.

The standard procedure of fluorescence in situ hybridization (FISH) analysis with metaphase chromosomes and DNA fibres was performed as described previously. ${ }^{12,13}$

\section{Results and discussion}

To check the validity of similarities between Notl-linking clone sequences and genes we selected clone NR-025. We sequenced an area of about $500 \mathrm{bp}$ surrounding this $\mathrm{N}$ otl site. The sequence displayed $51 \%$ identity over 35 amino acids with retinoblastoma binding protein 2 (RBBP2). ${ }^{14}$ No significant similarity was found based on nucleic acids $(64.8 \%$ identity in $108 \mathrm{bp}$ overlap). After extending the sequenced area to $1.2 \mathrm{~kb}$ we found two EST clones with overlapping sequences (AA729218 and AA232348). Using these two cDNAs and a combination of different methods, ie cDNA library screening, RT-PCR and in silico-cloning, we finally obtained the sequence of 7450 bp which includes 779 bp of $5^{\prime}$ genomic sequences and $6671 \mathrm{bp}$ of cDNA sequences (Accession No.AJ243706). This combined sequence encodes a predicted protein of 1681 amino acids (Figure1, 2). The protein displays a high level of amino acid identity to RBBP2 (see Figure2). In fact, in many extended regions they are completely identical. This is the reason why we called this gene RBBP2H1A ( $\mathrm{Rb}$ binding protein 2 homologue $1 \mathrm{~A}$ ). Nevertheless, nucleic acid identity is much less profound, in the best cases reaching $79-84 \%$.

Search with the amino acid sequences of the gene using ProfileScan at the ISREC server (see Materials and M ethods) revealed many potential domains (Figure1b). Among them the most obvious were three $\mathrm{Zn}$-finger domains (446-496 aa, 1313-1361 aa and 1621-1675 aa) and bipartite nuclear localization signals (1239-1256 aa and 1536-1553 aa). There are also many other motifs, like amidation sites, CAMP- and
cGMP-dependent protein kinase phosphorylation sites, casein kinasell phosphorylation sites, etc. We suggest that two methionine residues could correspond to the start codon: no. 1 in Figure2 (758 bp) and no. 61 (938bp). Located upstream are two predicted promoter sites (555-605 bp and $816-866 \mathrm{bp}$ ). Specific experiments must be conducted to determine which of them is the correct starting point but here we suggest that the first methionine is indeed the start. The basis for this is that we have found 5 ' cDNA clone (AA729218) starting at the $779 \mathrm{bp}$ and is thus located upstream of the predicted promoter for the second methionine. Numerous putative transcription factor binding signals can be found throughout the first $500 \mathrm{bp}$, eg for transcription factors Sp1 (122-129 bp), Pax-5 (209-232 bp), Oct-1 (255-262 bp), PU.1 (262-271 bp), Skn-1 (376-387bp) etc. Finding the real promoter can be a difficult task since sequences outside the conventional promoter can play a role in this function, for instance pertaining to exon sequences. ${ }^{15}$ On the other hand genes can also have multiple promoters. A predicted molecular weight of the longest polypeptide is $185 \mathrm{kDa}$.

We performed an analysis of the expression with CDNA clone AA232348 as a probe (1080-4510bp, see AJ243706), using MTN northern filters containing polyA ${ }^{+}$RNAs from 15 different human tissues (Clontech). Two bands of approximately $6 \mathrm{~kb}$ and $7 \mathrm{~kb}$ were found (Figure3). However, testis RNA most probably contains another band, $8.5 \mathrm{~kb}$ in size. The gene is expressed in all tested tissues but the level of the expression varies very significantly. The highest level of expression was detected in testis and the lowest in skeletal muscle. Moreover, the ratio between the two main bands also varied between tissues.

We have tested renal cell carcinoma (RCC) biopsies (four cases) and 10RCC cell lines. No noticeable changes in expression were detected (data not shown).

Using FISH, we assigned the genomic clone NR-025 to chromosomal band 1q32.1 (Figure4). To test the integrity of the NR-025 linking clone, we performed the fiber FISH. This experiment reveal ed the absence of any significant rearrangements and confirmed the integrity of the NR-025 (data not shown).

No significant deletions or rearrangements have been reported in the literature to be associated with this area. However, in the region another RB-binding protein, RBQ-3, was mapped by Saijo et al. ${ }^{16}$

After we started to clone this gene, Ueki et al ${ }^{17}$ published a paper that described a new method for cloning nucleartargeted proteins. Among other genes and gene fragments, they isolated a cDNA (AB015348) that contains the 3 ' end of the RBBP2H $1 \mathrm{~A}$. The homology starts from amino acid 1218 of RBBP $2 \mathrm{H} 1 \mathrm{~A}$. The AB015348 CDNA clone contain sa deletion of five amino acids at the extreme $3^{\prime}$ end of our gene (1595-1600 aa). Ueki et $\mathrm{al}^{17}$ have also shown that their candidate gene fragment is coding for a nuclear-targeted protein, allowing us to speculate that the complete gene 
A

\begin{tabular}{|c|c|c|c|c|c|c|c|c|c|c|c|c|c|c|c|c|c|}
\hline 1 & $T$ & $T C A$ & GTC & $T G T$ & $N A A$ & AGG & $A T A$ & $C C C$ & CCG & TTG & $T G A$ & $A T T$ & TCG & TAC & CTG & TAT & \\
\hline 47 & $G A C$ & $C A G$ & $A G A$ & $C A C$ & СTC & $A A A$ & TTC & $A \nexists A$ & ATG & $T C C$ & $A G A$ & $A \bar{A} T$ & $G A A$ & $C A C$ & $A G T$ & $A T C$ & 4 \\
\hline 95 & TTC & TTC & $C C T$ & $T G A$ & $G T A$ & $A C T$ & $C G C$ & $C T T$ & CTG & TAT & ATA & $A T T$ & GGG & GTG & $A A T$ & $G T T$ & 2 \\
\hline 143 & $C C A$ & $G C A$ & $A T G$ & $C C A$ & ACG & $G G C$ & TAG & CAT & CTA & CTC & $A G G$ & $C A G$ & $G T A$ & $C G C$ & CAG & $A C A$ & \\
\hline 191 & $T C T$ & $G A A$ & $T C T$ & TAA & TAC & CTC & GTC & GTC & $C C C$ & $C C T$ & $C A A$ & $T C T$ & GTC & $A C T$ & $A T G$ & $T C C$ & \\
\hline 239 & $T A A$ & $T T T$ & $T A T$ & TTC & CTA & $C C T$ & $T T G$ & CAT & $C C C$ & $T T C$ & CTC & $A T A$ & $A T T$ & ATC & $A G C$ & $A C C$ & \\
\hline 287 & $A C T$ & $G C C$ & TTA & $A T T$ & TAG & ATC & CTA & $T C A$ & TTC & CTT & GTC & $T G G$ & $A T T$ & $A T T$ & $T C A$ & $A G T$ & \\
\hline 335 & $C T T$ & $T T G$ & $C C C$ & СТC & $T C A$ & $A G T$ & CTG & $A C C$ & $T C G$ & $T C A$ & $T A G$ & $A C T$ & $G T T$ & $T G T$ & GGA & $T G A$ & \\
\hline 383 & TTA & $T T T$ & $T C C$ & CTG & $C A A$ & CAT & СTC & CAC & CTC & $C C A$ & ATA & GTC & $T C C$ & ATC & $A C T$ & $T A T$ & \\
\hline 431 & TAG & $C C A$ & $T G T$ & $G A A$ & $T T A$ & TGG & $C A A$ & $A T T$ & GCT & $T A A$ & $C C T$ & $A C C$ & $T A T$ & $G C C$ & $T C A$ & CTT & \\
\hline 479 & TTC & CTT & $A T T$ & GTA & $A A G$ & $T G G$ & $A A A$ & TAA & $G A G$ & $T A C$ & CTA & $T C T$ & $C A T$ & AGG & GTT & $G T G$ & \\
\hline 527 & $T G A$ & $T T A$ & ATC & $A T G$ & $C G A$ & $T G C$ & $A A T$ & TAG & $A A C$ & $A G T$ & $G C C$ & TAG & GAT & ATG & $A A A$ & GTG & \\
\hline 575 & $C C C$ & $C A A$ & $A A T$ & GTT & $A C G$ & $T T A$ & TTA & TTA & TTA & TTA & $C T A$ & CTA & TTG & $C C T$ & $T C T$ & $C A T$ & \\
\hline 623 & GAA & $C A T$ & GCT & $A T G$ & TGT & ATG & TTC & $A A G$ & CTG & TTA & $T C T$ & CTA & CTG & GAA & $A A T$ & $C C T$ & \\
\hline 671 & TCT & $C C G$ & $T T T$ & $C C A$ & $A T A$ & CTC & $T T T$ & $C C A$ & $A A A$ & $C C C$ & $C C T$ & $C C T$ & GGG & $T T T$ & TAC & $T G C$ & \\
\hline 1 & & & & & & & & & & & & & & M & G & L & \\
\hline 719 & $A C C$ & $T A C$ & $C A T$ & $T G T$ & GTA & $C T G$ & $A C G$ & $T G A$ & $T T G$ & $A A T$ & GAT & TGG & $C T T$ & ATG & $G G A$ & $C T G$ & \\
\hline 4 & I & L & $S$ & $\mathrm{~T}$ & $\mathrm{R}$ & L & $\mathrm{N}$ & $\mathrm{F}$ & $E$ & $R$ & A & G & $\mathrm{T}$ & L & I & $\mathrm{H}$ & \\
\hline 767 & $A T C$ & CTT & $T C T$ & $A C C$ & AGA & CTT & AAC & TTC & TTC & AGG & $\mathrm{GCA}$ & GGA & $\mathrm{ACT}$ & CTG & ATA & $\mathrm{CAT}$ & \\
\hline 20 & S & K & W & $S$ & I & K & V & G & S & $\mathrm{T}$ & $E$ & $T$ & L & $\mathrm{R}$ & I & $\mathrm{N}$ & \\
\hline 815 & $\mathrm{AGT}$ & AAA & TGG & TCA & ATA & AAA & GTT & GGC & TCA & $\mathrm{ACT}$ & GAA & $\mathrm{ACC}$ & CTA & AGG & ATA & AAC & \\
\hline 36 & S & I & $\mathrm{H}$ & $S$ & G & $\mathrm{R}$ & A & $\mathrm{R}$ & $\mathrm{P}$ & $\mathrm{R}$ & $P$ & $I$ & $\mathrm{R}$ & G & $\mathrm{R}$ & G & \\
\hline 863 & TCC & ATC & CAC & AGC & GGA & AGA & GCG & CGG & $\mathrm{CCC}$ & CGC & $\mathrm{CCG}$ & ATT & CGG & GGG & CGG & GGC & \\
\hline 52 & G & $\mathrm{E}$ & $\mathrm{P}$ & $\mathrm{R}$ & A & $E$ & G & G & $\mathrm{S}$ & M & G & V & G & W & D & $S$ & \\
\hline 911 & GGG & GAG & CCG & CGC & GCG & GAG & GGT & GGG & TCG & ATG & GGG & GTG & GGG & TGG & GAC & TCT & \\
\hline 68 & $\mathrm{~F}$ & S & $S$ & P & W & $\mathrm{R}$ & $\mathrm{R}$ & $\mathrm{R}$ & $\mathrm{R}$ & $\mathrm{T}$ & A & W & A & C & G & $E$ & \\
\hline 959 & TTT & $\mathrm{TCC}$ & TCG & CCG & TGG & CGG & CGG & AGG & CGC & ACG & GCG & TGG & GCT & TGC & GGC & GAG & \\
\hline 84 & $T$ & $S$ & $S$ & S & $\mathrm{E}$ & A & $E$ & K & A & $Q$ & G & A & A & V & A & $R$ & \\
\hline 1.007 & ACG & TCG & TCG & TCG & GAG & $\mathrm{GCT}$ & GAA & AAA & GCC & CAA & GGT & $\mathrm{GCT}$ & $\mathrm{GCC}$ & GTT & GCC & CGT & \\
\hline 100 & $\mathrm{~T}$ & $\mathrm{~T}$ & $\mathrm{R}$ & $\mathrm{T}$ & $\mathrm{C}$ & $\mathrm{C}$ & $\mathrm{C}$ & $S$ & $\mathrm{~S}$ & $\mathrm{R}$ & $\mathrm{V}$ & $\mathrm{C}$ & $\mathrm{T}$ & G & L & G & \\
\hline 1055 & $\mathrm{ACA}$ & $\mathrm{ACT}$ & CGG & $\mathrm{ACT}$ & TGC & TGT & TGC & TCG & AGC & CGC & GTC & TGC & ACG & GGT & CTC & GGA & \\
\hline 116 & $\mathrm{P}$ & S & G & A & $\mathrm{R}$ & $S$ & L & G & $\mathrm{P}$ & G & $\mathrm{A}$ & $\mathrm{H}$ & L & $\mathrm{R}$ & $\mathrm{L}$ & A & \\
\hline 1103 & CCG & AGC & GGA & GCT & CGC & AGC & $\mathrm{CTC}$ & GGT & $\mathrm{CCC}$ & GGA & GCC & CAC & СTT & CGC & СТC & GCC & \\
\hline 132 & L & A & $Q$ & P & A & V & I & $\mathrm{E}$ & A & & $\mathrm{T}$ & $\mathrm{T}$ & & $\mathrm{H}$ & $\mathrm{P}$ & G... & \\
\hline 151 & $\mathrm{CTT}$ & G & & & & & & & & & 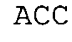 & $\mathrm{CA}$ & & $\mathrm{CAC}$ & $\mathrm{CCA}$ & GGC. & \\
\hline
\end{tabular}

B

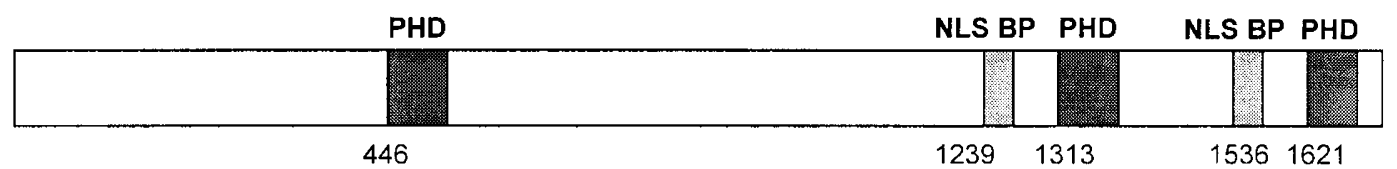

Figure 1 Structure of the RBBP2H1A gene A Nucleotide and amino acid sequences of the 5' end RBBBP2H1A. Upstream genomic sequences are in bold italic. The first three methionine residues are in bold. B Localization of zink-finger like PHD and bipartite nuclear targeting sequence (NLS BP) motifs in the RBBP2H1A.

isolated in the present study is also localized in the cell nucleus. During the preparation of the manuscript a new entry in the EMBL database was revealed. In March 1999 Vogt et al (AF087481) reported the sequence of a new RBBP2 homologous protein (RBBP2H 1 ) and noted that expression of this gene is differentially regulated during progression of malignant melanomas. The sequence of RBBP2H1 is very similar to the sequence of RBBP2H1A with small deletions/ insertions or changes involving ten amino acids. Two significant differences were revealed, however. Our sequence is missing amino acids $238-273$ of RBBP $2 \mathrm{H} 1$ and at the same time the starting methionine in their sequence is only the third in our sequence (137 aa). Thus, RBBP2H 1 is 101 amino acids shorter than RBBP2H1A described in the present study.

In May $1999 \mathrm{Lu}$ et $\mathrm{al}^{18}$ published the isolation and sequencing of a new gene PLU-1. This gene is identical to our gene starting from $1080 \mathrm{bp}$; thus our gene is 137 aa longer. 


\begin{tabular}{|c|c|c|}
\hline $\begin{array}{l}\text { rbbp } 2 \mathrm{~h} 1 \mathrm{a} \\
\text { rbbp2h1 } \\
\text { rbbp2 }\end{array}$ & 1 & $\begin{array}{l}\text { MGLILSTRLNFFRAGTLIHSKWSIKVGSTETLRINSIHSGRARPRP IRGRGGEPRAEGGS } \\
-\end{array}$ \\
\hline rbbp2h1a & 61 & MGVGWDSFSSPWRRRRTAWACGETSSSEAEKAQGAAVARTTRTCCCSSRVCTGLGPSGAR \\
\hline $\begin{array}{l}\text { rbbp } 2 \mathrm{~h} 1 \\
\text { rbbp2 }\end{array}$ & & 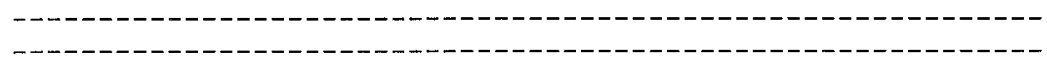 \\
\hline & & \\
\hline rbbp2h1a & 121 & SLGPGAHLRLALAQPA WMEAATTLHPGPRPALPLGGPGPLGEFLPPPECPVEE PSWEEFA \\
\hline rbbp2h1 & 1 & $--1-1$ \\
\hline rbbp2 & 1 & $-\cdots-M A G$ GPGEYAAE FVPPPECPVEE PSWEEFT \\
\hline rbbp2h1a & 181 & DPFAFIHKIRPIAE QTGICKVRPPPDWQPPFACD GDKLHFTPRIQRLNELEAQTRVKLNF \\
\hline rbbp2h1 & 44 & DPEAFIHKIRPIAEQTGICKVRPPPDWQPPFACDVDKLHFTPRIQRLNELEAQTRVKLNF \\
\hline rbbp2 & 31 & DPLSFI IRIRRIAEKTGICKIRPPKDWQPPFACEVKSFRFTPRVQRLNELEAMTRVRLDF \\
\hline rbbp2h1a & 241 & LDQIAKYWELQGSTLKIPHVERKILDLFQLNKLVAEE GGEAVVCKDRKWTKIATKMGEAP \\
\hline rbbp2h1 & 104 & LDQIAKYWELQGSTLKI PHVERKILDLFQLNKLVAEEGGFAVVCKDRKWTKIATKMGEAP \\
\hline rbbp2 & 91 & LDQIAKEWELQGSTLKIPVVERKILDLYALSKIVASKGGEEMVTKEKKWSKVGSRLGY LP \\
\hline rbbp2h1a & 301 & GKAVGSHIRGHYERILNPYNLFL SGD SLRCLQKPNLTIDTKDKEYKPHDIPQRQSVQPSE \\
\hline rbbp2h1 & 164 & GKAVGSH IRGHYERILNPYNLFLSGD SLRCLQKPNLTTDTKDKEYKPHD IPQRQSVQPSE \\
\hline rbbp2 & 151 & GKGTGS LLKSHYERIL YPYELE QSG VSLMGVQMPNL DLKEK---VEPEVLSTDTQTSPE- \\
\hline rbbp2h1a & 361 & TCPPARRAKRMRAE-- \\
\hline rbbp2h1 & 224 & TCPPARRAKRMRAERQSLAVLPRLECSGAILAHCNLRLLDSSNSSASASQAMNIKIE PEE \\
\hline rbbp2 & 207 & -- PGTRMNILPKR-- \\
\hline rbbp2h1a & 385 & TTEARTHNLRRRMGCPTPKCENEKEMKSS IKQE P IERKDY IVE-NEKEKPKSRSKK---A \\
\hline rbbp $2 \mathrm{~h} 1$ & 284 & TTEARTHNLRRRMGCPTPKCENEKEMRSSIKQEP IERKDYIVE-NEKEKPKSRSKK---A \\
\hline rbbp2 & 228 & GDVSRNTEIKKLQIFGAGPKVVGLAMGTKDKEDEVTRRRKVTNRSDAFNMQMRQRKGTLS \\
\hline rbbp2h1a & 441 & TNAVDLYVCLICGSGNDEDRLLLCDGCDDSYHTFCLIPPLHDVPKGDWRCPKCLAQECSK \\
\hline rbbp2h1 & 340 & TNAVDLYVCLLCGSGNDEDRLLLLCDGCDDSYHTFCLIPPLHDVPKGDWRCPKCLAQECSK \\
\hline rbbp2 & 288 & VNFVDLYVCMFCGRGNNEDKLLLCDGCDDSYHTFCLIPPLPDVPKGDWRCPKCVAEECSK \\
\hline rbbp2h1a & 501 & PQEAFGEEQAARDYTLRTFGEMADAFKSDYFMMPVHMVPTELVEKE FWRI \\
\hline rbbp2h1 & 400 & POEAFGFEQAARDYTLRTFGEMADAFKSDYFNMPVHMVPTELVEKE EWRLVSTIEEDVTV \\
\hline rbbp2 & 348 & PREAFGFEQA VREYTL QSFGEMAD NEKSDY FNMPVHMVPTELVEKE FWRLVSS IEEDVIV \\
\hline rbbp2h1a & 561 & EYGADIASKEFGSGFPVRDGKIKLSPEEEEYLDSGWNLNNMPVMEOSVLAH ITAD ICGMK \\
\hline rbbp2h1 & 460 & EYGADIASKEFGSGFPVRDGKIKLSPEEEEYLDSGWNLNNMPVMEQSVLAHITADICGMK \\
\hline rbbp2 2 & 408 & EYGAD ISSKDFGSGFPVKDGRRKI LPEEEEY ALSGWNLNNMPVLEQSVLAHINVDISGMK \\
\hline rbbp $2 \mathrm{~h} 1 \mathrm{a}$ & 621 & LPWLYVGMCF SSFCWHTEDHWSYS INYLHWGE PKTWYGV \\
\hline rbbp $2 \mathrm{~h} 1$ & 520 & LPWLYVGMCF SSFCWHIEDHWSYS INY LHWGE PKTWYGVPGYAAE QLENVMKKLAPELFV \\
\hline rbbp2 & 468 & VPWLYVGMCFSSFCWHIEDHWSYS INYLHWGEPKTWYGVP SHAAEOLEEVMRELAPELFE \\
\hline rbbp2h1a & 681 & SQPDLLHQLVTIMNPNTLMTHEVPVYRTNQCAGE FVITF PRAYHSGFNQGFNFAEAVNFC \\
\hline rbbp2h1 & 580 & SQPDLLHQLVTIMNPNTLMTHEVPVYRTNQCAGEFVITFPR VP-QWFN \\
\hline rbbp2 & 528 & SQPDLLHQLVTTMNPN VLMEH GVPVYRTNQCAGEFVVTEPRAYHSGENQGYNFAEAVNFC \\
\hline rbbp2h1a & 741 & TVDWLPLGRQCVEHYRLLHRYCVESHDEMICKMASKADVLDVVVASTVQKOMA IMIEDEK \\
\hline rbbp2h1 & 639 & TVDWLPLGROCVEHYRLLHRYCVFSHDEMICKMASKADVLDVVVASTVOKDMA TMIEDEK \\
\hline rbbp2 2 & 588 & TADWLP IGRQCVNHYRRLRR HCVFSHEELI FKMAADPE CIDV GLAAMV CKELTLMTEEE T \\
\hline rbbp2h1a & 801 & ALRE TVRKLGVIDSERMD FELLPDDERQCVKCKTTCEMSAISCSCKPGLLVCLHHVKELC \\
\hline rbbp2h1 & 699 & ALRE TVRKLGVID SERMD FELLLDDERQCVKCKTTCFMSA ISCS WKPGLLVCLHHVKELC \\
\hline rbbp2 & 648 & RLRESVVQMGVLMSEEEVEELVPDDERQCSACRTTCELSALTCSCNPERLVCL YHPTDLC \\
\hline & 861 & WVNEALEAKITKKKSLVSEKA \\
\hline $\mathrm{cbbp} 2 \mathrm{~h} 1$ & 759 & CPPYKYKLLRYRYTLDDLYPMMNALKLRAESYNEWPLNVNEALEAKITKKKSLVSEKA \\
\hline rbbp2 2 & 708 & PCPMOKKCLRYRY PLEDLPSLLYGVKVRAQSYDTWVSRVTEALSANENHKKDIIELRVML \\
\hline
\end{tabular}

Figure 2 Alignment of the deduced amino acid sequence of RBBP2H1A with sequences of RBBP2H1 [AF087481] and RBBP2. ${ }^{14}$ Grey boxes and underlined letters indicate identical and similar amino acid residues, respectively. 
rbbp2hla 921 EESEMKKFPDNDLIRHLRLVTQDAEKCASVAQOLINGKRQTRYRSGGGKSQNQLTVNELR rbbp2h1 819 EESEMKKFPDNDLLRHLRLVTQDAEKCASVAQQLINGKRQTRYRSGGGKSQNQITVNELR rbbp2 768 EDAE DRKYPENDL ERKLRDAVKEAE TCASVAQLLLSKKQKHRQSPDSGRTRTKLTVEELK

rbbp2h1a 981 QFVTQLYAIPCVLSQTPLLKDLLNRVEDFQQHSOKLISEETPSAAELQDLLDVSFEFDVE rbbp2h1 879 QFVTQLYALPCVL SQTPLLKDLTNRVEDFQQHSQKLLSEE TPSAAELQDLIDVSFEFDVE rbbp2 828 AFV COLFSLPCVISQARQVKNLIDDVEE F HERAQE AMMDE TP DSSKLQML IDMGSSL IVE

rbbp2h1a1041 LPQLAEMRIRLEQARWLEEVQQACIDPSSLTLDDMRRLIDLGVGLAPYSAVEKAMARLQE rbbp2h1 939 LPQIAEMRIRLEQARWLEEVQQACLDPSSLTLDDMRRL IDLGVGLAPYSAVEKAMARLQE rbbp2 888 LPEL PRLKQELQQARWLDEVRLTLSDPQQVTLD VMKKL ID SGVGLAP HHAVEKAMAELQE

rbbp $2 h 1 a 1101$ LLTVSEHWDDKAKSLIKARPRHSLNSLATAVKE IEEIPAYLPNGAALKDSVQRARDWLOD rbbp2h1 999 LLTVSEHWDDKAKSLLKARPRHSLNSLATAVKE IEEIPAYLPNGAALKDSVQRARDWLQD rbbp2 948 LLTVSERWEEKAKVCLQARPRHSVASLES TVNE AKNIPAELPNVLSLKEALQKAREWTAK

rbbp2h1a1161 VEGLQAGGRVPVIDTLIELVTRGRS IPVHLNSLPRLE TLVAEVQAWKECAVNTFLTENSP rbbp2h1 1059 VEGLQAGGRVPVIDTLIELVTRGRS IPVHLNSLPRLETLVAEVQAWKECAVNTFLTENSP rbbp2 1008 VEAIQSGSNYAYLECLESLSAKGRPIPVRLEALPQVESQVAAARAWRERTGRTFLKKNSS

rbbp $2 h 1$ a1221 YSLLEVLCPRCD IGLLGLKRKQRK-LKEPLPNGKKKSTKLESLSDLERAITESKETASAM rbbp2h1 1119 YSLIEVLCPRCD IGLLGLKRKQRK-LKEPLPNGKKKSTKLESLSDLERALTESKETASAM rbbp2 1068 HTLLQVLSPRTDIGVYGSGKNRRKKVKE LIEKEKEKDLDLEPLSDLEEGLEETRDTAMV

rbbp $2 \mathrm{~h} 1 \mathrm{a} 1280$ ATLGEARLREMEALQSLRLANEGKLLSPLQDVDIKICLCOKAPAAPMIQCELCRDAFHTS rbbp2h1 1178 ATLGEARLREMEALQSLRLANEGKLLSPLODVDIKICLCRKAPAAPMIQCELCRDAFHTS rbbp2 1128 AVFKE REQKE IEAMHSLRAAN LAKMTMVDR IEEVKFCICRKTASGFMLQCELCKDWFHNS

rbbp $2 \mathrm{~h} 1 \mathrm{a} 1340$ CVAVPSISQGLR--------IWLCPHCRRSEKPPLEKILPLLASLQRIRVRLPEGDALR rbbp2h1 1238 CVAVPSISQGLR---------IWLCPHCRRSEKPPLEKILPLIASLQRIRVRLPEGDALR rbbp2 1188 CVPLPKSSSQKKGSSWQAKEVKFLCP LCMRSRRPRLE TII SLI VSLQKLPVRLPEGEALQ

rbbp2h1a1391 YMIERTVNWQHRAQQILSS-_.

rbbp2h1 1289 YMIERTVNWQHRAQQLISS-.--_-_-

rbbp2 1248 CLTERAMSWQDRARQALATDELSSALAKLSVLSQRMVEQAAREKTEKI ISAELQKAAANP

rbbp2h1a1410 ---GNLKEVQD----RVGSGLLYSRWQASAG----------------QVSDTNKVS--rbbp2h1 1308 ---GNLKFVQD----RVGSGLLYSRWQPSAG-rbbp2 1308 DLQGHLPSFQQSAFNRVVSSVSSSPRQTMDYDDEETDSDEDIRETYGYDMKDTASVKSSS

rbbp2h1a1443 -------QPPGTTSESLPDDWDNRTSYLHSPFS-TGRSCIPLHG-

rbbp2h1 1341 -

rbbp2 1368 SLEPNLFCDEE IP IKSEEVTTHMWTAPS FCAEHAYSSASKSCSQVFFGKGSSTPRKQPRK

rbbp2h1a1479 ---------_--VSP-D--EVNELLMEAQLLQVSLPEIQELYQTLLAKPS PAQQTDRS rbbp2h1 1378 --.---_-----VSP----EVNELLMEA ALLQVSLPE IQELYQTLIAKPSPAQQTDRS rbbp2 1428 SPLVPRSLEPPVLELSP GAKAQLEELMMVGDLLEVSLDE TQHIWRILQATHPPSE--DRF

rbbp2h1a1521 SPVRPSS---EKNDCCRGKRDGINSLERKLLRRLEREGLSSERWERVKKMRTPKKKKIKL rbbp2h1 1420 SPVRPSS---EKNDCCRGKRDGINSLERKLLRRLEREGLSSERWERVKKMRTPKKKKKIKI rbbp2 1486 LHIMEDDSMEEKPLKVKGKDSSEKKRKRKLEKVEQLLFGEGKQKSKELKKMDKPRKKKLKI

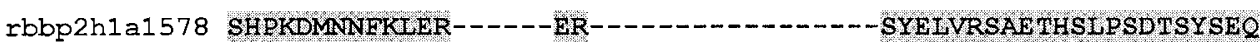
rbbp2h1 1477 SHPKDMNNFKLER------ER---_--------SYELVRSAETHSLPSDTSYSEQ rbbp2 1546 GADKSKKLNKLAKKLAKEEERKKKKEKAAAAKVELVKESTEKRREKKVLDIPSKYDWS GA

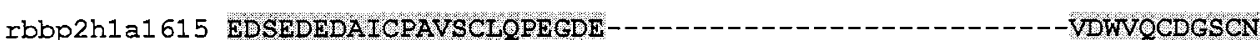

rbbp2h1 1514 EDSEDEDAICEAVSCLQPEGDE-_-_.

rbbp2 1606 EESDDENAVCAEPDCQRPCKDKGVVEVTKKREIKNISFKSVLCDCFSKKVDWVQCDGGCD

rbbp2h1a1648 QWFHQVCVGVSPEMAEKEDY ICVRCTVKDAPSRK

rbbp2h1 1547 QWFHQVCVGVSPEMAEKEDYICVRCTVKDAPSRK

rbbp2 1666 EWEHRVCVGVSPEMA--

Figure 2 Continued. 


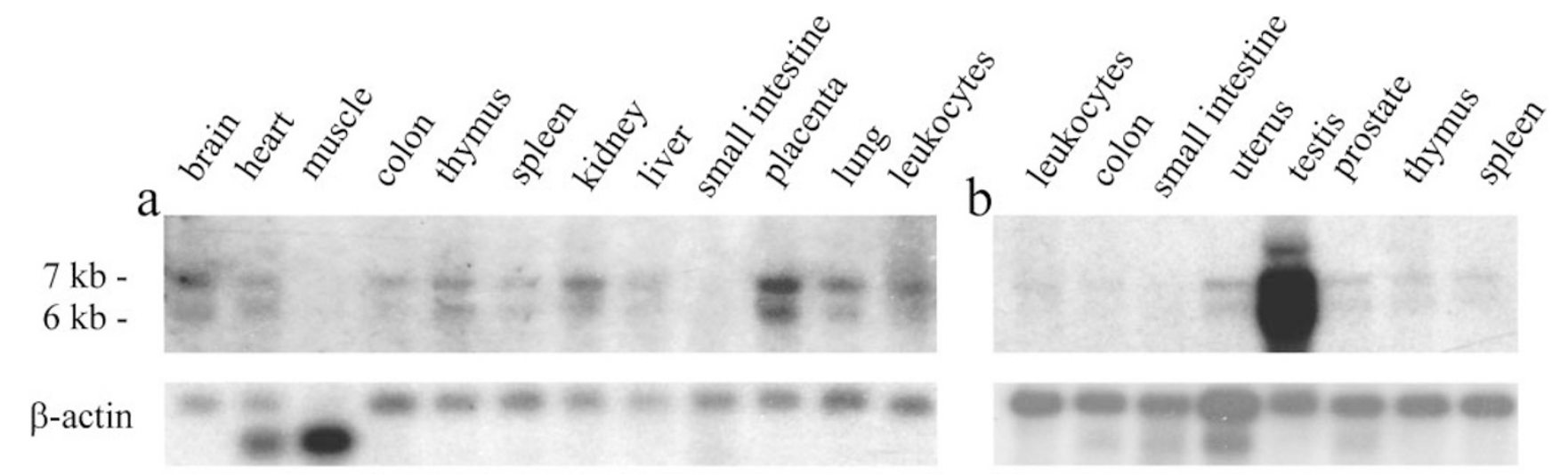
Figure 3 Hybridization of the RBBP2H1A to two Clontech northern blots a Human 12-Line MTN Blot I; b Human MTN BlotIV.
The lower panels show hybridization of the $\beta$-actin to the same filter.

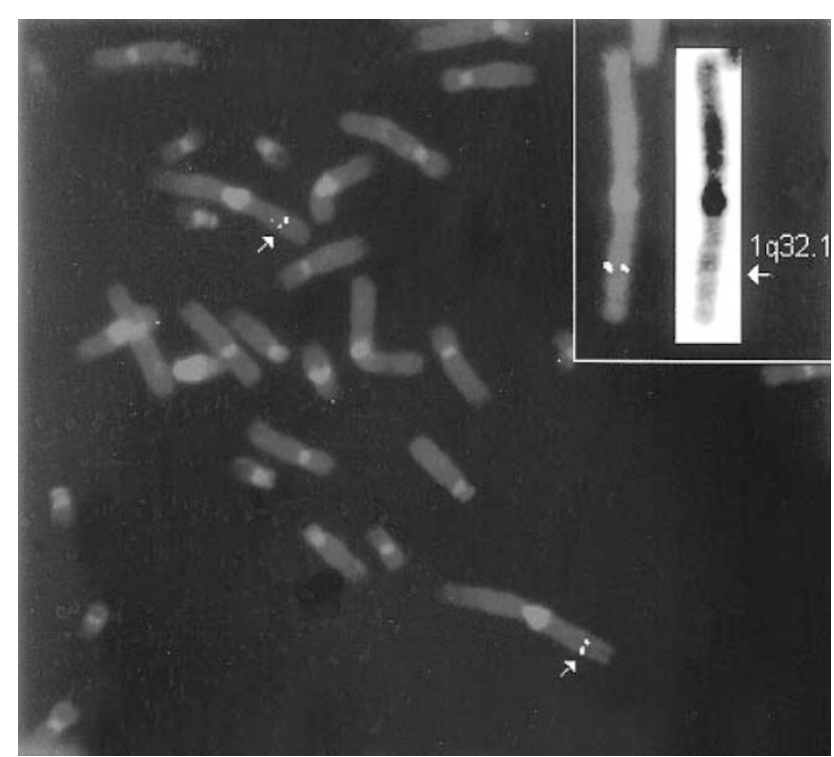

Figure 4 Localization of RBBP2H1A by FISH. Arrows indicate the position of RBBP2H1A.

Northern expression data are strikingly different for RBBP2H1A and PLU-1. Lu et al ${ }^{18}$ reported highly restricted expression of PLU-1 in adult tissues. PLU-1 showed high expression in testis and low levels of expression in placenta, ovary and tonsil. We detected two RNA bands ( $6 \mathrm{~kb}$ and $7 \mathrm{~kb}$ ) in all 15 tested normal adult tissues (in testis even 3RNA species were detected, additional $8.5 \mathrm{~kb}$ band is also seen). However, the level of expression varied very significantly in these tissues. In skeletal muscles and small intestine expression is hardly detected and in testis it is very strong. It is important to mention that we used as a probe a cDNA clone containing a majority of the RBBP $2 \mathrm{H} 1 \mathrm{~A}$ coding sequences including 5 ' part absent in the PLU-1 probe (1080-4510 bp). Lu et al used the PLU-1 probe containing mainly the $3^{\prime}$ untranslated region of the PLU-1 gene (4712-6636 bp of
RBBP2H1A). Furthermore we used northern blots with poly $A^{+}$RNA and Lu et al used total RNA for the northern blots.

The authors reported elevated expression of PLU-1 in breast cancer. As was mentioned above, we found no elevated expression in RCC biopsies and cell lines.

One explanation of all the results from different groups is that these genes represent alternatively spliced mRNAs of the same gene. Alternatively, these genes may represent closely related members of the same gene family. As the RBBP2H1A and PLU-1 were mapped to the same chromosomal band it is more likely that they represent splice variants of the same gene but further experiments are needed to resolve this question.

\section{Acknowledgements}

This work was supported by research grants from the Swedish Cancer Society, Pharmacia \& Upjohn, Karolinska Institute and Åke W iberg foundation. VIK was recipient of fellowship from the Concern Foundation in Los Angeles and the Cancer Research Institute in N ew York.

\section{References}

1 Zabarovsky ER, Kashuba VI, Zakharyev VM et al: Shot-gun sequencing strategy for long range genome mapping: first results. Genomics 1994; 21: 495-500.

2 Allikmets RL, Kashuba VI, Pettersson B et al: N otl linking clones as a tool for joining physical and genetic maps of the human genome. Genomics 1994; 19: 303-309.

3 Zabarovsky ER, Allikmets R, Kholodnyuk I et al: Construction of representative $\mathrm{Notl}$ linking libraries specific for the total human genome and for human chromosome3. Genomics 1994; 20: 312-316.

4 Kashuba VI, Gizatullin RZ, Protopopov Al et al: Analysis of Notl linking clones isolated from chromosome 3 specific libraries. Gene 1999; 239: 259-271.

5 Zabarovsky ER, Boldog F, Erlandsson R et al: New strategy for mapping the human genome based on a novel procedure for construction of jumping libraries. Genomics 1991; 11: 1030-1039.

6 Ichikawa H, Hosoda F, Arai Y, Shimizu K, Ohira M, Ohki M: A Notl restriction map of the entire long arm of human chromosome21. Nat Genet 1993; 4: 361-366. 
7 Hosoda F, Arai Y, Kitamura E et al: A complete N otl restriction map covering the entire long arm of human chromosome11. Genes to Cells 1997; 2: 345-357.

8 Bird AP: CpG islands as gene markers in the vertebrate nucleus. Trends Genet 1997; 3: 342-347.

9 Kashuba VI, Gizatullin RZ, Protopopov Al et al: Notl linking/ jumping clones of human chromosome 3 : mapping of the TFRC, RAB7 and HAUSP genes to regions rearranged in leukemia and deleted in solid tumors. FEBS Letters 1997; 419: 181-185.

10 Gish W, States DJ: Identification of protein coding regions by database similarity search. Nat Genet 1993; 3: 266-272.

11 Altschul SF, Gish W, Miller W, Myers EW, Lipman DJ: Basic local alignment search tool. J Mol Biol 1990; 215: 403-410.

12 Pinkel D, Straume T, Gray JW: Cytogenetic analysis using quantitative, high sensitivity, fluorescence hybridization. Proc Natl Acad Sci USA 1986; 83: 2934-2938.

13 Protopopov Al, Gizatullin RZ, Vorobieva NV et al: High resolution FISH mapping of $50 \mathrm{~N}$ otl linking clones homologous to genes and cDNAs on human chromosome3. Chromosome Res 1996; 4: 443-447.
14 Fattaey AR, Helin K, Dembski MS et al: Characterization of the retinoblastoma binding proteins RBP1 and RBP2. Oncogene 1993; 8: 3149-3156.

15 Leprini A, Gherzi R, Vecchi E, Borsi L, Zardi L, Siri A: Rat tenascin$R$ gene: structure, chromosome location and transcriptional activity of promoter and exon 1. Cytogenet Cell Genet 1998; 83: $115-123$.

16 Saijo $M$, Sakai $Y$, Kishino $T$ et al: Molecular cloning of a human protein that binds to the retinoblastoma protein and chromosomal mapping. Genomics 1995; 27: 511-519.

17 Ueki N, Oda T, Kondo M, Yano K, Noguchi T, Muramatsu M: Selection system for genes encoding nuclear-targeted proteins. Nat Biotechnol 1998; 16: 1338-1342.

$18 \mathrm{Lu}$ PH, Sundquist K, Baeckstrom D et al: A novel gene (PLU-1) containing highly conserved putative DNA/Chromatin binding motifs is specifically up-regulated in breast cancer. JBC 1999; 274: 15633-15645. 